\title{
Transition Radiation of Charged Particle in the Modulated Anisotropic Magnetodielectric Filling of the Waveguide
}

\author{
E. GEVORKYAN* \\ Plekhanov Russian University of Economics, 117997 Moscow, Russia
}

\begin{abstract}
The main results of theoretical investigations related to the questions of transition radiation of a charged particle in a regular waveguide with periodically modulated anisotropic magnetodielectric filling, which were carried out by the author during several years, are summarized in this report. It is assumed that the charged particle moves perpendicular to the waveguide axis with a constant velocity. The analytical expressions for the transverseelectric and transverse-magnetic fields in a first approximation with respect to the small indexes of modulation are found. The energies of transition radiation in the region of "weak" interaction between the radiation wave and the modulated filling in the case of rectangular waveguide are calculated. The analysis of obtained results shows that the fields in the waveguide represent the set of space harmonics with different amplitudes and in the expressions for the energy of transition radiation, unlike for the case of the unmodulated filling, a member proportional to the modulation indexes of the first degree, is added.
\end{abstract}

DOI: 10.12693/APhysPolA.134.174

PACS/topics: TE and TM electromagnetic waves, regular waveguide, charged particle, transition radiation, modulated anisotropic magnetodielectric filling, Mathieu-Hill type differential equation

\section{Introduction}

In [1] the problem of transition radiation of charged particle, moving perpendicular to the axis of the waveguide with anisotropic magnetodielectric filling was solved. In this work the similar problem in the case, when the anisotropic magnitodielectric filling of the waveguide is modulated in space by a periodic law, is considered. This problem represents certain interest from the point of view of development of the theory and possible applications of periodic media in various fields of microwave electronics [2-8].

\section{Statement of the problem and its solution}

Let a charged particle with a charge $q$ propagates through the waveguide along the $O x$ axis with a constant velocity $\boldsymbol{v}=\{v ; 0 ; 0\}$ perpendicularly to the axis of the waveguide $o z$ and intersects the waveguide surface at the points $A_{1}\left(x_{1}, y_{0}, 0\right)$ and $A_{2}\left(x_{2}, y_{0}, 0\right)$. We shall assume, that the anisotropic magnetodielectric filling of the waveguide is modulated along $z$ axis according to the harmonic law and the dielectric permittivity and magnetic permeability of the filling have the form

$$
\widehat{\varepsilon}=\left(\begin{array}{ccc}
\varepsilon_{1} & 0 & 0 \\
0 & \varepsilon_{1} & 0 \\
0 & 0 & \varepsilon_{2}(z)
\end{array}\right), \quad \widehat{\mu}=\left(\begin{array}{ccc}
\mu_{1} & 0 & 0 \\
0 & \mu_{1} & 0 \\
0 & 0 & \mu_{2}(z)
\end{array}\right) \text {, }
$$

where

$$
\begin{aligned}
& \varepsilon_{2}(z)=\varepsilon_{2}^{0}\left(1+m_{\varepsilon} \cos k_{0} z\right), \\
& \mu_{2}(z)=\mu_{2}^{0}\left(1+m_{\mu} \cos k_{0} z\right),
\end{aligned}
$$

\footnotetext{
*e-mail: gevor_mesi@mail.ru
}

$\varepsilon_{1}=$ const, $\mu_{1}=$ const, $\varepsilon_{2}^{0}=\left.\varepsilon_{2}(z)\right|_{m_{s}=0}, \mu_{2}^{0}=$ $\left.\mu_{2}(z)\right|_{m_{\mu}=0}, m_{\varepsilon} \ll 1$ and $m_{\mu} \ll 1$ are the small indexes of modulation, $k_{0}$ is the wave number of modulation wave. As is known, the charge density and the current density in this case are described using Dirac $\delta$ function and have the form $[1,5]$

$$
\begin{aligned}
& \rho=q \delta(x-v t) \delta\left(y-y_{0}\right) \delta(z), \\
& j=j_{x}=q v \delta(x-v t) \delta\left(y-y_{0}\right) \delta(z) .
\end{aligned}
$$

The transverse-electric (TE) and transverse-magnetic (TM) fields in the waveguide, as in our previous works [1-6], we shall describe by means of longitudinal components of the magnetic vector $H_{z}(x, y, z, t)$ and of the electric vector $E_{z}(x, y, z, t)$. The wave equations for $\tilde{H}_{\omega z}=\mu_{2}(z) H_{\omega z}$ and $\tilde{E}_{\omega z}=\varepsilon_{2}(z) E_{\omega z}$ can be obtained from Maxwell equations taking into account Eq. (1). Calculations lead to the following wave equations in Fourier representation

$$
\begin{aligned}
& \Delta_{\perp} \tilde{H}_{\omega z}+\frac{\mu_{2}(z)}{\mu_{1}} \frac{\partial^{2} \tilde{H}_{\omega z}}{\partial z^{2}}+\varepsilon_{0} \mu_{0} \varepsilon_{1} \mu_{2}(z) \omega^{2} \tilde{H}_{\omega z}= \\
& \mu_{2}(z) \frac{\partial j_{\omega}}{\partial y}, \\
& \Delta_{\perp} \tilde{E}_{\omega z}+\frac{\varepsilon_{2}(z)}{\varepsilon_{1}} \frac{\partial^{2} \tilde{E}_{\omega z}}{\partial z^{2}}+\varepsilon_{0} \mu_{0} \mu_{1} \varepsilon_{2}(z) \omega^{2} \tilde{E}_{\omega z}= \\
& \quad \frac{\varepsilon_{2}(z)}{\varepsilon_{1} \varepsilon_{0}} \frac{\partial \rho_{\omega}}{\partial z},
\end{aligned}
$$

where

$$
\begin{aligned}
& j_{\omega}=\frac{1}{\sqrt{2 \pi}} q \delta\left(y-y_{0}\right) \delta(z) \mathrm{e}^{\mathrm{i} \omega x / v}, \\
& \left.\rho_{\omega}=\frac{q}{\sqrt{2 \pi} v} \delta\left(y-y_{0}\right)\right) \delta(z) \mathrm{e}^{\mathrm{i} \omega x / v} .
\end{aligned}
$$

$\Delta_{\perp}=\partial^{2} / \partial x^{2}+\partial^{2} / \partial y^{2}$ is the two-dimensional Laplace operator. The solutions of the Eqs. (4) and (5) will be sought in the form 


$$
\begin{aligned}
\tilde{H}_{\omega z} & =\sum_{n=0}^{\infty} \tilde{H}_{n}(z) \widehat{\psi}_{n}(x, y), \\
\tilde{E}_{\omega z} & =\sum_{n=0}^{\infty} \tilde{E}_{n}(z) \psi_{n}(x, y),
\end{aligned}
$$

where the orthonormal eigenfunctions $\widehat{\psi}_{n}(x, y), \psi_{n}(x, y)$ and the corresponding eigenvalues $\widehat{\lambda}_{n}, \lambda_{n}$ of the second and first boundary-value problems for the cross-section of the waveguide (the Neuman and Dirichlet problems) satisfy to known Helmholtz equations with appropriate boundary conditions (see (10) and (11) from [5]). The analytical expressions for the transverse components of the TE an TM fields are obtained from the Maxwell equations. In Fourier representation they have the form

$$
\begin{aligned}
& \boldsymbol{H}_{\omega \tau}=\frac{1}{\mu_{1}} \sum_{n=0}^{\infty} \widehat{\lambda}_{n}^{-2} \frac{\mathrm{d} \tilde{H}_{n}(z)}{\mathrm{d} z} \nabla \widehat{\psi}_{n}(x, y), \\
& \boldsymbol{E}_{\omega \tau}=-\mathrm{i} \omega \mu_{0} \sum_{n=0}^{\infty} \widehat{\lambda}_{n}^{-2} \tilde{H}_{n}(z)\left[\boldsymbol{z}_{0} \nabla \widehat{\psi}_{n}(x, y)\right],
\end{aligned}
$$

for TE field and

$$
\begin{aligned}
& \boldsymbol{H}_{\omega \tau}=-\mathrm{i} \omega \varepsilon_{0} \sum_{n=0}^{\infty} \lambda_{n}^{-2} \tilde{E}_{n}(z)\left[\boldsymbol{z}_{0} \nabla \psi_{n}(x, y)\right], \\
& \boldsymbol{E}_{\omega \tau}=\frac{1}{\varepsilon_{1}} \sum_{n=0}^{\infty} \lambda_{n}^{-2} \frac{\mathrm{d} \tilde{E}_{n}(z)}{\mathrm{d} z} \nabla \psi_{n}(x, y),
\end{aligned}
$$

for TM field, where $\nabla=\boldsymbol{i}(\partial / \partial x)+\boldsymbol{j}(\partial / \partial y)$ is the twodimensional Hamilton operator (nabla), $\boldsymbol{z}_{0}$ is the unit vector along the $O z$ axis, index $\tau$ indicates transverse components.

By substituting (8) and (9) into the (4) and (5) and having made some transformations in view of (2), (3), (6) and (7) for $\tilde{H}_{n}(z)$ and $\tilde{E}_{n}(z)$ we obtain the following equations

$$
\begin{aligned}
& \frac{\mathrm{d}^{2} \tilde{H}_{n}(z)}{\mathrm{d} z^{2}}+\widehat{\chi}_{n}^{2} \tilde{H}_{n}(z)=-\frac{q \mu_{1}}{\sqrt{2 \pi}} \delta(z) \widehat{B}_{n}, \\
& \frac{\mathrm{d}^{2} \tilde{E}_{n}(z)}{\mathrm{d} z^{2}}+\chi_{n}^{2} \tilde{E}_{n}(z)=\frac{q}{\sqrt{2 \pi} v \varepsilon_{0}} \delta^{\prime}(z) B_{n},
\end{aligned}
$$

where

$$
\begin{aligned}
& \widehat{B}_{n}=\left.\int_{x_{1}}^{x_{2}} \frac{\partial \widehat{\psi}_{n}(x, y)}{\partial y}\right|_{y=y_{0}} \mathrm{e}^{\mathrm{i} \omega x / v} \mathrm{~d} x, \\
& {\widehat{\chi})_{n}^{2}}^{2}=\frac{\mu_{1}}{\mu_{2}^{0}}\left[\left(\widehat{\chi}_{n}^{0}\right)^{2}+m_{\mu} \widehat{\lambda}_{n}^{2} \cos k_{0} z\right], \\
& \left(\widehat{\chi}_{n}^{0}\right)^{2}=\varepsilon_{0} \mu_{0} \varepsilon_{1} \mu_{2}^{0} \omega^{2}-\widehat{\lambda}_{n}^{2}, \\
& B_{n}=\left.\int_{x_{1}}^{x_{2}} \psi_{n}(x, y)\right|_{y=y_{0}} \mathrm{e}^{\mathrm{i} \omega x / v} \mathrm{~d} x, \\
& \chi_{n}^{2}=\frac{\varepsilon_{1}}{\varepsilon_{2}^{0}}\left[\left(\chi_{n}^{0}\right)^{2}+m_{\varepsilon} \lambda_{n}^{2} \cos k_{0} z\right], \\
& \left(\chi_{n}^{0}\right)^{2}=\varepsilon_{0} \mu_{0} \mu_{1} \varepsilon_{2}^{0} \omega^{2}-\lambda_{n}^{2} .
\end{aligned}
$$

By substituting (14) and (15) into Eqs. (12) and (13), by introducing a new variable $s=k_{0} z / 2$ and by decomposing in Fourier series the coefficients of $\tilde{H}_{n}(z)$ and $\tilde{E}_{n}(z)$, while limiting to the first three space harmonics, we obtain the following inhomogeneous Mathieu-Hill type differential equations

$$
\begin{aligned}
& \frac{\mathrm{d}^{2} \tilde{H}_{n}(s)}{\mathrm{d} s^{2}}+\sum_{k=-1}^{1} \widehat{\theta}_{k}^{n} \mathrm{e}^{2 \mathrm{i} k s} \tilde{H}_{n}(s)= \\
& -\frac{q \mu_{1}}{\sqrt{2 \pi}} \delta(s) \widehat{B}_{n}, \\
& \frac{\mathrm{d}^{2} \tilde{E}_{n}(s)}{\mathrm{d} s^{2}}+\sum_{k=-1}^{1} \theta_{k}^{n} \mathrm{e}^{2 \mathrm{i} k s} \tilde{E}_{n}(s)= \\
& \frac{q}{\sqrt{2 \pi} v \varepsilon_{0}} \delta^{\prime}(s) B_{n},
\end{aligned}
$$

where

$$
\begin{gathered}
\widehat{\theta}_{0}^{n}=\frac{4 \mu_{1}\left(\widehat{\chi}_{n}^{0}\right)^{2}}{k_{0}^{2} \mu_{2}^{0}}, \quad \widehat{\theta}_{ \pm 1}^{n}=\frac{2 \mu_{1} \widehat{\lambda}_{n}^{2}}{k_{0}^{2} \mu_{2}^{0}} m_{\mu}, \\
\theta_{0}^{n}=\frac{4 \varepsilon_{1}\left(\chi_{n}^{0}\right)^{2}}{k_{0}^{2} \varepsilon_{2}^{0}}, \quad \theta_{ \pm 1}^{n}=\frac{2 \varepsilon_{1} \lambda_{n}^{2}}{k_{0}^{2} \varepsilon_{2}^{0}} m_{\varepsilon} .
\end{gathered}
$$

The solutions of the corresponding homogeneous equations will be sought in the form

$$
\begin{aligned}
& \tilde{H}_{n}(s)=\mathrm{e}^{\mathrm{i} \widehat{\mu}_{n} s} \sum_{k=-1}^{1} \widehat{C}_{k}^{n} \mathrm{e}^{2 \mathrm{i} k s}, \\
& \tilde{E}_{n}(s)=\mathrm{e}^{\mathrm{i} \mu_{n} s} \sum_{k=-1}^{1} C_{k}^{n} \mathrm{e}^{2 \mathrm{i} k s},
\end{aligned}
$$

where the characteristic numbers $\widehat{\mu}_{n}, \mu_{n}$ and the amplitudes $\widehat{C}_{k}^{n}, C_{k}^{n}$ are the unknown quantities.

Substitution of (20) into the homogeneous equations results in the dispersion equations with respect to $\widehat{\mu}_{n}$, $\mu_{n}$ and in a system of equations with respect to $\widehat{C}_{k}^{n}, C_{k}^{n}$.

By solving the resulting equations in the region of "weak" (not resonant) interaction between the radiation wave and the modulation wave $\left(\hat{\theta}_{0}^{n}>1, \quad \theta_{0}^{n}>1\right)$, for $\widehat{\mu}_{n}, \mu_{n}$ and $\widehat{C}_{k}^{n}, C_{k}^{n}$ in the first approximation on the small indexes of modulation we get

$$
\begin{aligned}
& \widehat{\mu}_{n}^{2} \cong \widehat{\theta}_{0}^{n}, \quad \widehat{C}_{ \pm 1}^{n} \cong \frac{\widehat{\theta}_{1}^{n} \widehat{C}_{0}^{n}}{4\left(1 \pm \sqrt{\bar{\theta}_{0}^{n}}\right)}, \\
& \mu_{n}^{2} \cong \theta_{0}^{n}, \quad C_{ \pm 1}^{n} \cong \frac{\theta_{1}^{n} C_{0}^{n}}{4\left(1 \pm \sqrt{\theta_{0}^{n}}\right)},
\end{aligned}
$$

where $\bar{C}_{k}^{n}$ and $C_{k}^{n}$ can be found from the condition of normalization. The particular solutions of the inhomogeneous wave equations (16) and (17) will be sought by the method of variation of constants. The calculations in view of the radiation condition (no waves propagating to the radiation source) 
lead to the following expression for the TE and TM fields of transition radiation of a charged particle

$$
\begin{aligned}
& \tilde{H}_{n}(z)=\frac{\mathrm{i} \xi_{n}}{\widehat{C}_{0}^{n} \widehat{\mu}_{n} k_{0}} \sum_{k=-1}^{1} \widehat{C}_{k}^{n} \cos \frac{k_{0}\left(\widehat{\mu}_{n}+2 k\right) z}{2}, \\
& \tilde{E}_{n}(z)=\frac{k_{0} \zeta_{n}}{2 C_{0}^{n}} \sum_{k=-1}^{1} C_{k}^{n} \sin \frac{k_{0}\left(\mu_{n}+2 k\right) z}{2}
\end{aligned}
$$

where

$$
\xi_{n}=-\frac{q \mu_{1}}{\sqrt{2 \pi}} \widehat{B}_{n}, \quad \zeta_{n}=\frac{q}{\sqrt{2 \pi} \varepsilon_{0} v} B_{n} .
$$

The expressions (23) and (24) show that TE and TM fields of the transition radiation of charged particle in its motion perpendicular to the axis of the waveguide with periodically modulated anisotropic magnetodielectric filling is a sum of spatial harmonics with different amplitudes. The amplitude of the zeroth harmonic $(k=0)$ is not dependent on the modulation indexes and the amplitudes of the plus and minus first harmonics $(k= \pm 1)$ are proportional to the modulation indexes in the first order.

\section{Energies of transition radiation.}

\section{Results for the case of rectangular waveguide}

The energies of transition radiation of particle moving along its trajectory from $x_{1}$ to $x_{2}$ we shall find with the help of the value of the braking force $q E_{\omega x}$ acting on the particle via the field created by the particle. In the region of weak interaction between the modulation wave and the radiation wave the calculations with regard of (10), (11), (18), (19), (21), (22), (23), (24) and (25) in the first approximation, with respect to indexes of modulation, lead to the following expressions

$$
\begin{aligned}
& S_{n}^{(\mathrm{TE})}=\frac{\mu_{0} k_{0}^{2} \sqrt{\mu_{2}^{0} \mu_{1}} q^{2}}{4 \pi \bar{\lambda}_{n}^{2}} \\
& \times \operatorname{Re} \int_{0}^{\infty}\left(1+\frac{\mu_{1} \bar{\lambda}_{n}^{2}}{k_{0}^{2} \mu_{2}^{0}-4 \mu_{1}\left(\bar{\chi}_{0}^{n}\right)^{2} m_{\mu}}\right) \frac{\omega}{\bar{\chi}_{0}^{n}}\left|\widehat{B}_{n}\right|^{2} \mathrm{~d} \omega, \\
& S_{n}^{(\mathrm{TM})}=\frac{k_{0} q^{2}}{2 \pi \varepsilon_{0} \sqrt{\varepsilon_{1} \varepsilon_{2}^{0}} \lambda_{n}^{2} v^{2}} \\
& \quad \times \operatorname{Re} \mathrm{i} \int_{0}^{\infty}\left(1+\frac{3 \varepsilon_{1} \lambda_{n}^{2}}{k_{0}^{2} \varepsilon_{2}^{0}-4 \varepsilon_{1}\left(\chi_{n}^{0}\right)^{2}} m_{\varepsilon}\right) \omega\left|B_{n}\right|^{2} \mathrm{~d} \omega,
\end{aligned}
$$

where from the regions of integration are excluded the frequencies which satisfy the conditions

$$
k_{0}^{2} \mu_{2}^{0}-4 \mu_{1}\left(\bar{\chi}_{0}^{n}\right)^{2}=0, \quad k_{0}^{2} \varepsilon_{2}^{0}-4 \varepsilon_{1}\left(\chi_{n}^{0}\right)^{2}=0 .
$$

If in (26) and (27) we consider the limit of $m_{\mu} \rightarrow 0$ and $m_{\varepsilon} \rightarrow 0$, we shall receive the expressions for the energies of transition radiation of a charged particle in the case of unmodulated filling of the waveguide. Let us consider the case of a rectangular waveguide, the walls of which are defined by the equations $x=0, y=0, x=a, y=b$. By performing the integration with respect to variable $x(0 \leq x \leq a)$ in the expressions for $\widehat{B}_{n}$ and $B_{n}$ (see (14) and (15)), taking into account that the eigenfunctions $\left(\widehat{\psi}_{n}(x, y), \psi_{n}(x, y)\right)$ and eigenvalues $\left(\widehat{\lambda}_{n}, \lambda_{n}\right)$ are expressed by the formulas (18) and (19) from [1], for $S_{n, m}^{\mathrm{TE}}$ and $S_{n, m}^{\mathrm{TM}}$ we get

$$
\begin{aligned}
S_{n, m}^{\mathrm{TE}} & =\frac{\mu_{0} \sqrt{\mu_{2}^{0} \mu_{1}} q^{2} \pi n^{2} \delta_{n} \delta_{m} k_{0}^{2} \sin ^{2} \frac{\pi n}{b} y_{0}}{a b^{3} v^{2} \bar{\lambda}_{n, m}^{2}} \\
& \times \operatorname{Re} \int_{0}^{\infty}\left(1+\frac{\mu_{1} \bar{\lambda}_{n, m}^{2}}{k_{0}^{2} \mu_{2}^{0}-4 \mu_{1}\left(\bar{\chi}_{0}^{n, m}\right)^{2}} m_{\mu}\right) \\
& \times \frac{\omega^{3}}{\bar{\chi}_{0}^{n, m}} \frac{\sin ^{2}\left(\frac{\pi m}{a}-\frac{\omega}{v}\right) \frac{a}{2}}{\left[\left(\frac{\pi m}{a}\right)^{2}-\frac{\omega^{2}}{v^{2}}\right]^{2} \mathrm{~d} \omega,} \\
S_{n, m}^{\mathrm{TM}} & =\frac{8 q^{2} \pi m^{2} k_{0} \sin ^{2} \frac{\pi n}{b} y_{0}}{\varepsilon_{0} \sqrt{\varepsilon_{1} \varepsilon_{2}^{0}} a^{3} b v^{2} \lambda_{n, m}^{2}} \\
& \times \operatorname{Rei} \int_{0}^{\infty}\left(1+\frac{3 \varepsilon_{1} \lambda_{n, m}^{2}}{k_{0}^{2} \varepsilon_{2}^{0}-4 \varepsilon_{1}\left(\chi_{n, m}^{0}\right)^{2}} m_{\varepsilon}\right) \\
& \times \frac{\sin ^{2}\left(\frac{\pi m}{a}-\frac{\omega}{v}\right)^{\frac{a}{2}} \omega \mathrm{d} \omega,}{\left[\left(\frac{\pi m}{a}\right)^{2}-\frac{\omega^{2}}{v^{2}}\right]^{2}}
\end{aligned}
$$

where $\delta_{i}=2, i \neq 0, \delta_{0}=1$. As can be seen from (29) and (30), when the condition $n y_{0} / b=k(k=0,1,2, \ldots ; k \leq n)$ holds, in radiation there are no corresponding modes with the first index $n$. From (29) and (30) it also follows that at $n=0, S_{0, m}^{\mathrm{TE}}=0$, while at $m=0, S_{n, 0}^{T M}=0$. We also note that when the condition $\left(\frac{\pi m}{a}-\frac{\omega}{v}\right) \frac{a}{2}=l \pi(l$ is a nonzero integer) holds, the energies of transition radiation are zero.

\section{Conclusions}

In conclusion we note that the results obtained above allow to calculate the energies of transition radiation in the region of "strong" interaction between the modulation wave and radiation wave and to find some peculiarities of radiation in this region. Note, that the frequency range of the strong interaction is determined from the conditions (28), when the second terms in the brackets under the integrals in (26) and (27) or in (28) and (29) are meaningless.

\section{References}

[1] E.A. Gevorkyan, Optics and Spectros. 119, 286 (2015).

[2] E.A. Gevorkyan, Radiotekhnika i Elektronika 53, 565 (2008).

[3] E.A. Gevorkyan, IntechOpen 2011, pp. 267-284. 
[4] E.A. Gevorkyan, Proc. of ICEAA'12, Cape Town, South Africa 2012, pp. 877-879.

[5] E.A. Gevorkyan, Uspekhi Sovremennoy Radioelektroniki 3, 29 (2006).

[6] E.A. Gevorkyan, Electromagnitnie volni i elektronnie sistemi 14, 65 (2009).
[7] E.A. Gevorkyan, in: Proc. of 16th MMET'16, Lviv, Ukraine 2016, p. 334.

[8] E.A. Gevorkyan, O.V. Tatarnikov, in: Proc. of XXIst DIPED'16, Tbilisi, Georgia 2016, p. 17. 